\title{
Recurrent Prostate Acinar Adenocarcinoma
}

National Cancer Institute

\section{Source}

National Cancer Institute. Recurrent Prostate Acinar Adenocarcinoma. NCI Thesaurus.

Code C153570.

The reemergence of prostate acinar adenocarcinoma after a period of remission. 